\title{
"Networks in Cognitive Systems and Biomedicine: Cerebral Processes, Models and Mathematical Tools Design"
}

\author{
DIEGO COSMELLI ${ }^{1 *} \&$ ADRIAN G. PALACIOS ${ }^{1 \#}$
}

\begin{abstract}
${ }^{1}$ Instituto de Sistemas Complejos de Valparaíso, Subida Artillería \# 470, Cerro Artillería, Valparaíso,
* Escuela de Psicología, Pontificia Universidad Católica de Chile (PUC), Vicuña Mackenna 4860, Macul, Santiago de Chile \& Departamento de Psiquiatría, PUC, Marcoleta 391, $2^{\circ}$ Piso, Santiago de Chile

\# Centro de Neurociencia de Valparaíso, Facultad de Ciencias, Universidad de Valparaíso. Gran Bretaña 1111, Playa Ancha, Valparaíso.
\end{abstract}

\begin{abstract}
Convergence of clinical, empirical, methodological and theoretical approaches aimed at understanding the relation between brain function and cognition, is by now standard in most if not all academic programs in the area of Cognitive Science. This confederation of disciplines is one of the liveliest domains of inquiry and discussion into some of the most fundamental -and historically resilient- questions human beings have posed themselves. The contributions gathered in this special issue of Biological Research, directly inspired by the ongoing work at the Instituto de Sistemas Complejos de Valparaiso and the December 2006 CONICYT-INSERM-SFI workshop "Networks in Cognitive Systems / Trends and Challenge in Biomedicine: From Cerebral Process to Mathematical Tools Design", Chile, represent an explicit invitation to the reader to dive deeper into this fascinating terrain.
\end{abstract}

Key terms: Cognitive Sciences, Brain Dynamics, Mind-Brain.

\section{CONTEXT AND MOTIVATION}

Between August and November 2005 the Institute for Complex Systems of Valparaiso (ISCV) organized a series of seminars on the theme of "Complexity and Cognitive Sciences". This cycle of talks was a first attempt at bringing together national researchers involved in one way or another with the question of how the nervous system is related to cognition, and how complex system tools could be of relevance to advancing our understanding of this relation. One of the defining characteristics of these seminars was their strong discussion- and student-oriented approach to promote critical questioning of the way different researchers were tackling their respective problems. Indeed, thanks to the enthusiasm of participants, both from the students and from speakers, discussion flourished and several heated debates had to be continued after the seminar was formally over.

The success of this first approach encouraged the authors (who coordinated that activity) to go a step further and consider the possibility of an international meeting in Valparaiso where some specific questions that had been outlined during the seminars could be explored in more detail. In particular, we were interested in further understanding the role of different modeling strategies in the study of cognition; we wanted to go from the biomedical and methodological issues to

* Corresponding Author: Diego Cosmelli, email: dcosmelli@uc.cl 
the more conceptual and theoretical questions, again with a special focus on the nervous system.

These problems have become a major concern for the neuroscientific community in the last decades. The development of brain imaging methods, ranging from the morphological to the functional, has opened an invaluable window of observation into the structure and workings of the brain. Nevertheless, the question of how the huge amount of data and results obtained are interpreted and put into the context of adapted cognition remains at a fundamentally correlative stage. Generative models are scarce and theoretical accounts that link brain function to perception or consciousness still suffer fundamental shortcomings (Cosmelli \& Thompson 2007). It is obvious by now to anyone working on these issues that no single discipline will suffice to provide satisfactory answer, and that a radically interdisciplinary approach is necessary.

During 2006, and thanks to a growing relation between Chilean researchers and the Santa Fe Institute (SFI) for Complex Systems, USA, the possibility of coorganizing an SFI residency month at the ISCV became a reality. Mathematical, ecological, sociological, economical and neuroscientific discussions succeeded throughout December 2006 at the ISCV. This special issue of Biological Research is the product of the international CONICYTINSERM-SFI workshop "Networks in Cognitive Systems / Trends and Challenge in Biomedicine: From Cerebral Process to Mathematical Tools Design" that took place in that context, and which aimed at bringing together methodological, empirical and theoretical approaches to the problem of cognition.

In the remainder of this editorial introduction we will briefly outline the different contributions in the context of the three main themes that were developed throughout the workshop: methodological, empirical and theoretical. Nevertheless, likewise throughout the workshop, a recurring theme was precisely that such distinctions cannot be so clear cut, so this ordering is mainly formal and the reader is invited to look for convergence among the different approaches. We will then present two additional contributions, which were selected by the speakers among the student presentations, as those being of the highest level. These papers were invited for submission to this special number and have been commented by leading researchers in the corresponding fields. As we will see, the first of these contributions takes the form of a critical review of an important yet quite neglected theme in cognitive neuroscience, namely the problem of death, its relation to brain function and the limits of consciousness (but see Laureys 2005 for a notable exception). The second is a short critique of the relation between neural synchrony and consciousness, a very invogue approach to the problem of perception which sometimes is accepted all too easily.

\section{CONTRIBUTIONS}

\subsection{Methodological Issues}

A major issue in cognitive neuroscience is to be able to complement functional data from brain activity with precise anatomical information of the underlying structures. Moreover this can have enormous clinical applications. A promising approach to this question is the study of diffusion signals in Magnetic Resonance Imaging. In their contribution Steren Chabert and colleagues tackle the problem of diffusion signals and their possible interpretations. The diffusion coefficient has been shown to depend on cell concentration, membrane permeability, and cell orientation through white matter fibers, as well as a number of physiological parameters. The authors show that diffusion signals depend mainly on the restriction of water displacement through membranes, extra-cellular impediments and tissue heterogeneity. They highlight how these crucial factors have to be taken into account in order to generate valid interpretations both for biological and clinical questions.

On a more basic research oriented question, Lachaux and colleagues approach the question of relating brain activity to 
given cognitive states with a novel proposal: one should consider the interest of using exploratory sessions like those used in animal research -where varied stimuli are presented and neuronal response can be monitored online- with human beings. Being able to do this requires a considerable effort in terms of online signal analysis of the data that is being obtained by EEG, MEG or some other electrophysiological method. The authors develop a neurofeedback-based approach call "Brain TV" as a way of monitoring in real time the state of neuronal populations in human beings in multiple frequency bands. They illustrate the potentiality of this original approach with an intracranial recording session obtained from an implanted epileptic patient.

The last of our methodological contributions has a similar objective in mind, but tackles it from a different point of view, using offline sophisticated statistical techniques. Besserve and colleagues present us with classification algorithms that are capable of distinguishing specific brain states during ongoing EEG and MEG data with better than $95 \%$ accuracy, for data segments as short as 1 second. In their contribution the authors review the rationale behind these emerging approaches and then present their own highly optimized algorithm. It is important to note that the authors have in mind the possible applications to brain computer interfaces, a major trend in both basic and clinical research, which could shed substantial light onto the question of the neuronal basis of cognitive processes.

\subsection{Empirical Issues:}

The Cognitive Sciences are strongly dependent on theoretical paradigms. In his contribution, Maldonado reviews three main hypotheses (models) regarding the problem of neuronal coding in perception. The first is based on the cardinal activity of few cells that constitute the neuronal correlate of perception; the second stresses the importance of population coding distributed throughout brain areas; the third, known as the temporal correlation hypothesis, stresses activity from distributed neuronal populations as relevant correlates of perception but, importantly, incorporates the need of dynamic neural synchronization on a millisecond time scale to single out and coordinate relevant object features. But, as the author argues, it is not enough to stay on the theoretical level so he proposes that a different take on experimental paradigms is also needed if we are to study a brain that is inherently dynamic. Maldonado argues for the importance of free-viewing paradigms in the study of perception, and he offers important insights into the practical problems of using these approaches.

On a converging line Basile deals with another dynamical effect, namely individual variability, that is rarely taken into account but that can also affect substantially the way we understand functional mapping in the brain. The author presents evidence showing high subject-to-subject variability in attention-related (non-sensory) tasks. He also presents an alternative to brute-force averaging in order to disclose reproducible individual patterns. Basile closes his contribution with a discussion of what he deems has critical implications to functional mapping: the need to abandon group averaging of task-related cortical activity. This, he claims, can deeply challenge actual clinical interpretations of focal lesion consequences, functional reorganization, and neurosurgical planning.

While being awake and visually perceiving the world might be considered the standard context for the study of cognition, it is necessary not to forget that a crucial part of brain functioning is associated to the time when we sleep. Peirano and Algarin argue for the study of sleep as no longer a passive rest state for the brain. Indeed, Rapid Eye Movement (REM) sleep stage appears as an active condition of intense cerebral activity. Moreover, as the authors show, large amounts of sleep in early life suggest that sleep may play a critical role in brain maturation. At that point in development brain connectivity is high but specific functions are not fully in place -in terms of Edelman's theory brain complexity is still 
low. Peirano and Algarin propose that REM sleep would provide an endogenous source of activation, critical precisely for both the structural and functional maturation of the central nervous system.

\section{3.Theoretical Issues}

Brain complexity depends on the total amount of neural connectivity, on the number of subsets of functionally and well establish neural nets (e.g. visual, olfactory brain areas) as well the properties to integrate sensory input and differentiate it from the actual internal brain states allowing the emergence of unique conscious states (Edelman \& Tononi, 2000). Recently, an important effort in cognitive brain sciences has been the development of mathematical tools dealing with the recording of large-scale neuronal activity. In this context, it is important to have clear models against which data can be contrasted as well as explicit predictions generated. In their contribution, Goles and Palacios take a historical perspective on neural networks and discuss the advantages and limitations of the Artificial Neural Net (ANN) model. The main objective of their contribution is to discuss if and how these models can help understanding the functioning of simple brain circuits in particular, and brain neural complexity in general.

On a similar model-oriented vein, David presents a highly original contribution that seeks to put together two theoretical corpuses that on a first look appear not to deal with similar questions. The author presents in detail the mathematical foundations of a recently developed modeling strategy in brain imaging: Dynamic Causal Modeling (DCM). He then argues that with minimal modifications this approach is entirely compatible with autopoietic theory as developed by the Chilean school of Humberto Maturana and Francisco Varela. Interestingly, David shows how this might actually work beyond the theoretical considerations by presenting a compelling example with intracortical recordings of an epileptic patient.

On a more abstract approach, the last of our contributions deals with the emerging crosstalk between biologically inspired computing and cognitive science. Cosmelli and collaborators argue that these domains share a number of commonalities that might be of interest for both disciplines, especially when we see what has happened in the history of programming paradigms. After reviewing three major trends in the study of cognitive systems -computational cognitivism, connectionism and embodiment- the authors present a brief overview of the development of programming paradigms. They claim that the tendency to abstraction in latter is mirrored in natural biological systems, whereby novel organizations provide novel domains of interaction and increasing autonomy. The authors present and discuss three lines along which such convergence might be relevant for the study of cognition in both natural and artificial systems.

\section{ACKNOWLEDGMENTS}

The authors are grateful to the Departamento de Relaciones Internacionales of CONICYT (DRIC) who granted the necessary support to bring this project to reality. We encourage the reader to revise the historical note by Xavier Altamirano and Cecilia Velit of the DRIC in this special issue. DC and AGP are partially supported by PBCT-CONICYT ACT45

\section{REFERENCES}

COSMELLI D and THOMPSON D (2007) "Embodiment or envatment? Reflections on the bodily basis of consciousness". In John Stewart, Olivier Gapenne, and Ezequiel di Paolo, eds., Enaction: Towards a New Paradigm for Cognitive Science, MIT Press

LAUREYS S (2005) Science and society: death, unconsciousness and the brain. Nature Reviews Neuroscience 6: 899-909

EDELMAN GM and TONONI G (2000). A Universe of Consciousness: How Matter Becomes Imagination. Basic Books, New York, NY 